\title{
Picosecond Laser Chemistry in Supersonic Jet Beams
}

\author{
AHMED H. ZEWAIL $\uparrow$ \\ Arthur Amos Noyes Laboratory of Chemical Physics, California Institute of \\ Technology, Pasadena, CA 91125, USA
}

In this review we describe the development and the applications of the picosecond-jet technique, which utilizes a picosecond laser and a supersonically-cold jet beam of large molecules. The applications include studies of coherence (quantum beats), photodissociation, isomerization, and partial solvation. The results emphasize the role of intramolecular energy redistribution, and provide evidence for intramolecular threshold effect for rates vs. excess molecular energy. Simple theory for this redistribution of energy among certain modes in molecules is given, and comparison with RRKM calculation is also made to assess the nature of the statistical behavior for the redistribution.

\section{INTRODUCTION}

Understanding vibrational energy redistribution and bond breakage in isolated large molecules require knowledge and direct measurement of the unimolecular rates involved in these processes. In the abscence of thermal congestion, one can, in principle, selectively excite the molecule into a given vibrational state $m$ at a given energy $E$ and measure the rate described by $k(m, E)$. Hence, tests of statistical and nonstatistical behaviors in isolated molecules can be made. The existence of nonstatistical behavior for certain $m$ modes at a given energy is crucial for successful mode-selective laser chemistry.

Over the past three years we have been examining these selective rate processes using the technique of picosecond-jet spectroscopy ${ }^{1,2}-$ the picosecond laser does the selective excitation and allows for direct measurement of $k(m, E)$ by resolving the spectra in real time, while the jet beam expansion removes the thermal congestion since the

† Camille and Henry Dreyfus Foundation Teacher-Scholar. 
molecules in the beam are vibrationally and rotationally cold. With this technique we studied a number of chemical and photophysical processes which include:

1) Coherence and energy redistribution in isolated large molecules (anthracene, stilbene). ${ }^{1-3}$

2) Quantum beats and Zeeman effect probing of radiationless processes in pyrazine. ${ }^{4}$

3) Intramolecular trans-cis isomerization of stilbene in the beam.,

4) Intramolecular hydrogen bonding ${ }^{6}$ in methyl salicylate and related systems.

5) Chromophore-selective excitation. ${ }^{7}$

6) Bond breakage and vibrational energy redistribution of partially-solvated molecules in the jet beam. ${ }^{8,9}$

Here, we shall focus on (1) the description of the technique and the time resolution, (2) the results obtained for stilbene isomerization, and vibrational energy transfer, and (3) new results regarding bond breakage and energy redistribution in isoquinoline at different stages of solvation, by water, alcohol, ... etc., in the jet.

$$
=\mathrm{N}: \cdots\left(\mathrm{H}_{2} \mathrm{O}\right)_{n} \quad n=1,2 \ldots
$$

Finally, comparison with statistical theories, like the RRKM, will be made to access the degree of energy redistribution and its influence on the dynamics.

\section{THE PICOSECOND LASER-MOLECULAR BEAM APPARATUS}

The arrangement we used for interfacing the picosecond laser to the molecular beam (or free-jet) is schematically shown in Figure 1. The laser is a synchronously pumped, dye laser system whose coherence width, time and pulse duration were characterized ${ }^{10}$ by the SHG autocorrelation technique. The pulse width of these lasers is typically 1-2 ps, or 15 ps, when a cavity dumper is used. For detection, one of three techniques can be used:

1) Time-correlated single photon counting (dispersed fluorescence) using a fast photomultiplier (or microchannel plate) - this way we achieved a resolution of $\sim 100 \mathrm{ps}$ (HWHM) without deconvolution. 

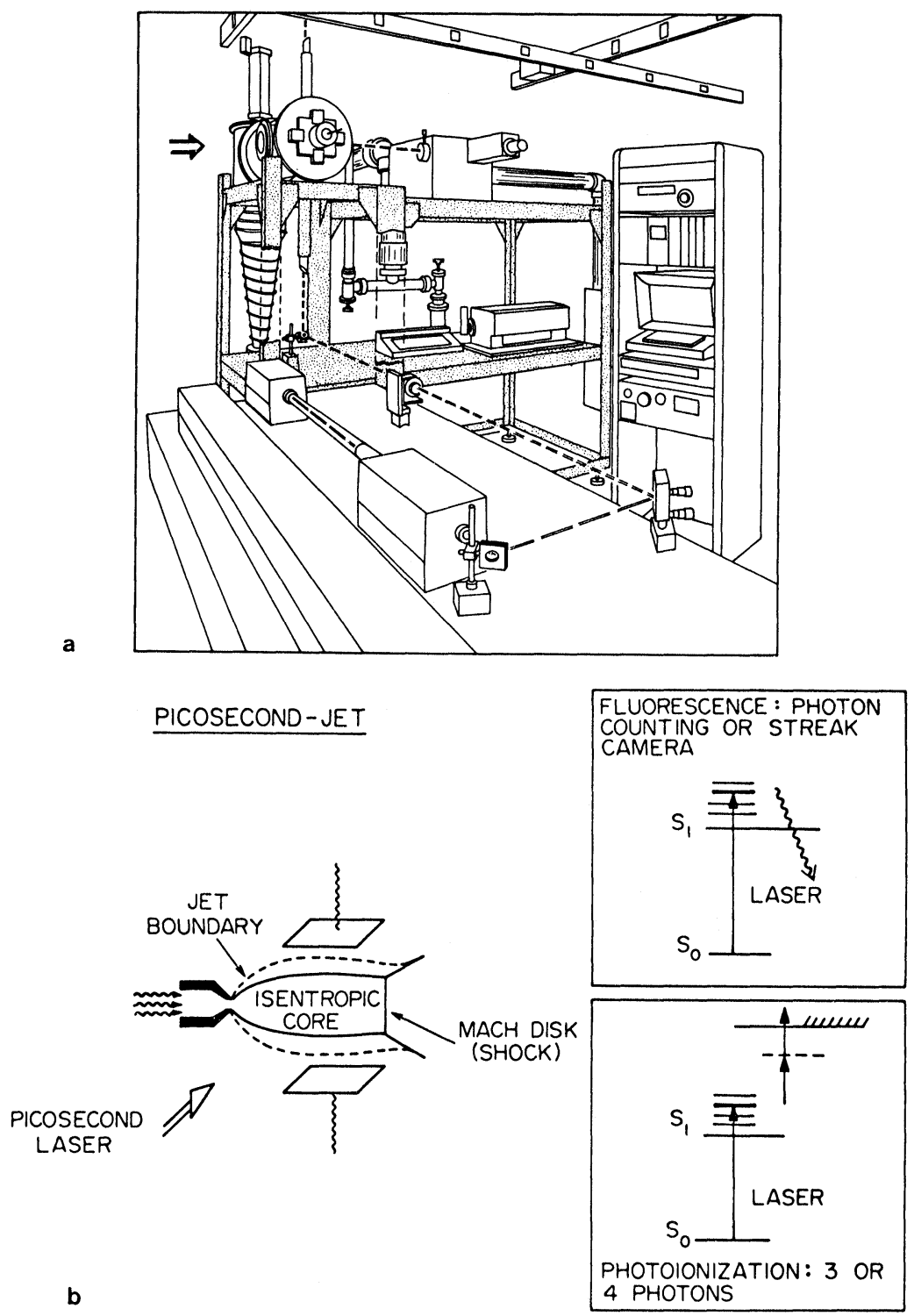

FIGURE 1 (a) The laboratory arrangement for the picosecond/molecular jet beam experiments; the arrow indicates the region where the picosecond pulse interrogates the molecular beam. (b) An outline of the jet expansion and detection methods. 
With convolution, one obtains, if required, the $30-50$ ps time constants of single exponential decays.

2) Streak-Camera-a synchroscan streak camera gives a resolution of better than $10 \mathrm{ps}$ with a repetition rate of up to $200 \mathrm{MHz}$.

3) Multiphoton ionization-we are developing this technique (see Figure 1) to obtain the short-time behavior, being limited by the pulse width of the laser ( 1 to $15 \mathrm{ps}$ ). This is applied to the stilbene isomerization problem (see Figure 1).

Finally, the actual laser bandwidth was varied by using a combination of intracavity filters and etalons. In all these experiments, the vibrational and rotational temperatures were typically $20-40 \mathrm{~K}$ and $1 \mathrm{~K}$, respectively, depending on the molecular beam condition.

\section{COHERENCE AND ENERGY REDISTRIBUTION}

Coherence in isolated large molecules can be probed if quantum beats are observable. Beats will be observed only if the homogeneous width, which relates to the rate of energy redistribution, is less than the energy separation between the states exhibiting the coherence (or interference) effects. The molecules we examined for this purpose are anthracene and stilbene.

The spectra of anthracene in a gas bulb at $\sim 480 \mathrm{~K}$ reveal very little intensity of quasi-sharp lines $\left(I_{s}\right)$ and a large background intensity that is very broad $\left(I_{b}\right)$ or diffuse in nature. When anthracene is jet-cooled, the $I_{s} / I_{b}$ varies dramatically depending on the excess vibrational energy in $S_{1}$. As shown in Figure 2, this ratio varies from 100 or more at zero excess energy to $10^{-2}$ or less at $5600 \mathrm{~cm}^{-1}$; the spectra in the high excess energy $\left(E_{x}\right)$ region are very similar to the bulb spectrum.

At about $1400 \mathrm{~cm}^{-1}$ of excess energy in anthracene, we observed quantum beats in the dispersed fluorescence with a large modulation depth. The beats are very sensitive to the excess energy and to the fluorescence detection wavelength. (This work is now completed by Wm. R. Lambert and P. M. Felker of this laboratory, and will be published elsewhere.) For stilbene, the quantum beats appeared at a number of different $E_{x}$. Clearly, the observation of beats in these large molecules is related to the coherence of the vibrational-rotational states excited, a point that we shall discuss later. The beats, 


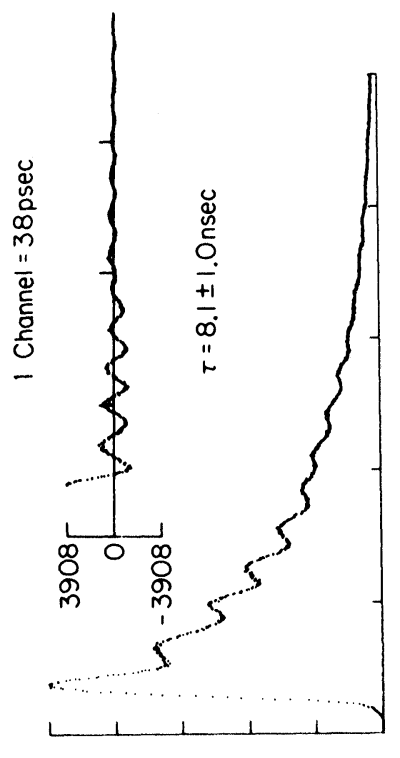

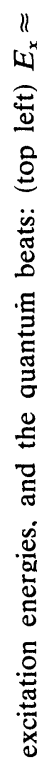

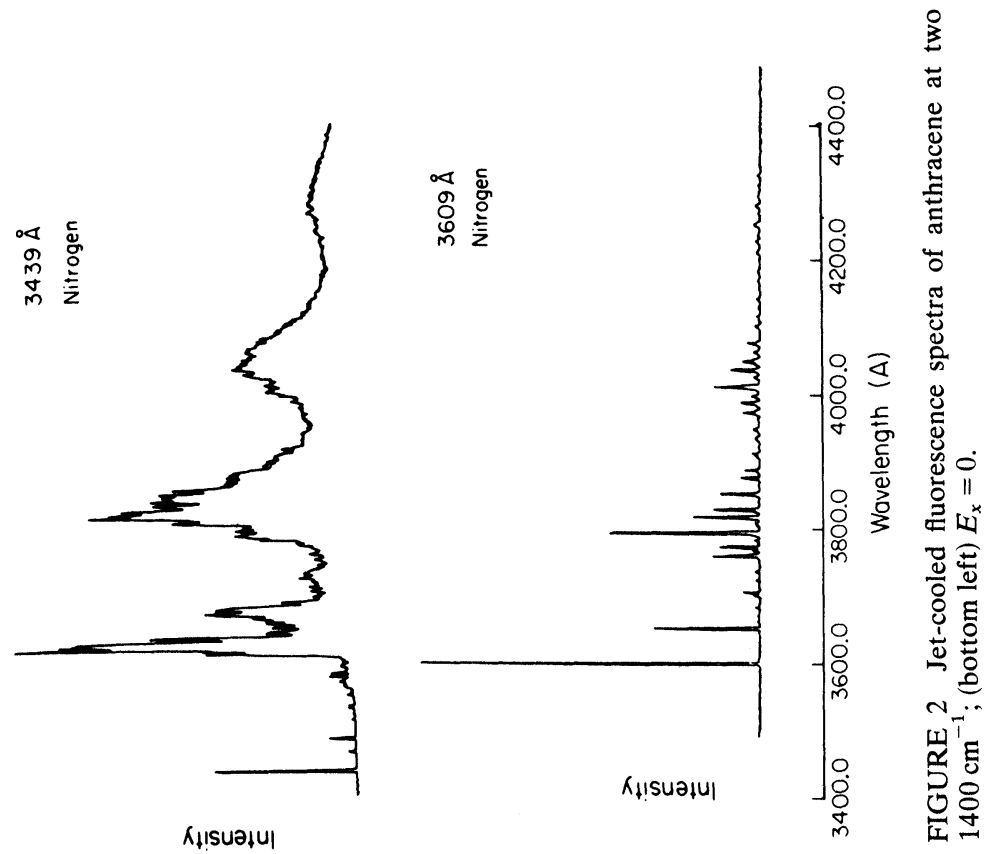


due to rovibronic states, in anthracene are not expected to be sensitive to magnetic fields in contrast with beats due to coupling between electronic states, one of them is triplet. In a recent work we reported ${ }^{4}$ on the Zeeman effect on quantum beats in pyrazine, and on the importance of beats to unravel the coupling of singlet and triplet rotational levels. In a more recent work by Kommandeur's group, ${ }^{11}$ the high resolution frequency spectra of pyrazine was observed and compared with our Fourier-transform beat spectra; very good agreement was found.

As it is well-known, one cannot observe quantum beats unless the separation between energy states is larger than or comparable to the width of these states (or bunch of states). In anthracene, the results of Lambert et al. ${ }^{1}$ must therefore indicate that the rovibronic states prepared by the laser pulse (at the measured excess vibrational energy) are not severely overlapping. In fact, a limit on the homogeneous width is now available; $<500 \mathrm{MHz}$. The apparent width of the excitation spectra is, of course, much larger (implying ps relaxation) than this width, but, in principle, by using single-mode laser excitation we should be able to resolve bands that correlate with the beat frequency, especially in the resonance fluorescence region. In this region there is no complexity due to the presence of many levels in the final state of the emission.

The interesting findings in anthracene are: (1) the uniqueness of the observation of beats at certain excess energy, and the sensitivity of the observation to detection wavelengths; (2) the shortening of lifetimes at higher $E_{x}$. These lifetimes do not vary drastically in magnitude even though the dispersed fluorescence broadens in a dramatic way at higher $E_{x}$ values. Actually, the "broad" feature of the dispersed fluorescence is not a real continuum. This suggests that the final state of the emission could be highly structured. To clarify the involvement of the final state (or states) in the observed spectra, Keelan ${ }^{12}$ is examining the effect of density-of-states in the initial and final manifolds, and couplings on the overall redistribution.

The reason that these beats are sensitive to excitation and detection energies is clear. For the excitation process, we must form linear combinations of these rovibronic states in order to see the interference (coherent) effects. The laser source, which was varied with respect to coherence time and width, has pulse substructure with each of the "noise" spikes of the substructure having large enough bandwidth to 
span the excited states to form the superposition. If the damping is faster than the period of the beats, as it will be at high excess energies, then no beats will be observed. In the decay process, on the other hand, one must have branching transitions, i.e., a common level to the emitting states. If the oscillator strength of one of the beating levels to the final state is very different, the modulation depth will be altered. The modulation depth of anthracene beats varied depending on the detection wavelength, and the modulation frequency changed when the rotational temperature varied.

What causes the coupling between the vibrational states? Two possibilities exist. The first is a simple anharmonic coupling between the vibrational modes which will conserve the rotational angular momentum between manifolds. The second is the Coriolis coupling which depends on $J$ and $K$ of the rotational structure. The simplicity of the beat pattern in anthracene, and the involvement of the rotational states in the redistribution (see the article by Parmenter in this issue) ${ }^{13}$ will be discussed in the forthcoming paper of Lambert and Felker, which assigns the rovibrational states involved. The important point here is that independent of the nature of the states involved, it is clear that some strongly coupled levels in the molecule at certain excess vibrational energies are coherent for subnanoseconds, as evident from the data on anthracene and stilbene. More recently, similar findings have been found for molecules in their ground electronic state but also with excess vibrational energy. ${ }^{14}$

\section{ISOMERIZATION IN THE JET AND $k(m, E)$}

The isomerization process

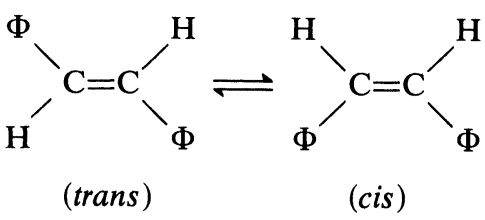

involves some torsional vibrational modes for the twisting to finalize. This is a classic problem in chemistry, and much work has been done in solutions ${ }^{15}$ and more recently under gas phase isolated molecule 
conditions. ${ }^{16}$ We have shown that the jet-cooled spectra ${ }^{3}$ of $t$-stilbene displays the isolated molecule low-frequency modes which are optically-active. Furthermore, the observed rates measured ${ }^{3}$ as a function of excess energy (Figure 3) exhibit a threshold at $\sim 1200 \mathrm{~cm}^{-1}$. This threshold value is in good agreement with the estimated barrier height for isomerization from $S_{1}$ in solution. The jet studies clearly indicate the absence of thermal energy influence on rates, and the involvement of the redistribution in the isomerization. An interesting question arising from these studies is: What is the origin of this threshold effect? In what follows some general ideas are presented.

The initial temperature of the molecule is very low and certainly is much lower than $\hbar \omega / k$ of totally symmetric modes. We may divide the modes of the molecule into those which are optically active (predominately totally symmetric or "relevant" $R$ ) and those which are not excited directly by the laser (bath modes $B$ ). This division of the system-bath interactions accounts for dephasing and energy relaxation by $T_{2}$ and $T_{1}$ time constants as discussed elsewhere. ${ }^{17,18}$

The redistribution of excitation of $R$ to $B$ will have a threshold depending on the number of quanta that can be populated in $B$ (density of states) and the degree of coupling. Invoking these lowfrequency modes in the redistribution suggests the use of restricted density of states since high frequency modes are not efficiently populated. It is interesting that in all molecules we studied such modes do exist, and it is perhaps a universal character of these large molecules to involve these modes in the redistribution (in anthracene, e.g., the change in rates around the threshold is not as dramatic as in the other molecules which enjoy more of these low-frequency modes). The "suppression" of the activity of these modes by the Shpolskii effect in matrices may be the reasons for obtaining sharp spectra, or in our language, in enhancing the $I_{s} / I_{b}$ ratio. The question is now: Knowing the mode structure, can we predict theoretically the energy threshold effect and the values of $k(m, E)$ ?

Here, we shall outline an approach that is simple, but perhaps too simple. For the redistribution, we will define a redistribution parameter ("temperaiure") which describes the rate according to the following equation:

$$
k=A e^{-E_{0} / k T_{\text {eff }}}=A e^{-\bar{n} E_{0} / E_{x}}
$$


PICOSECOND/JET

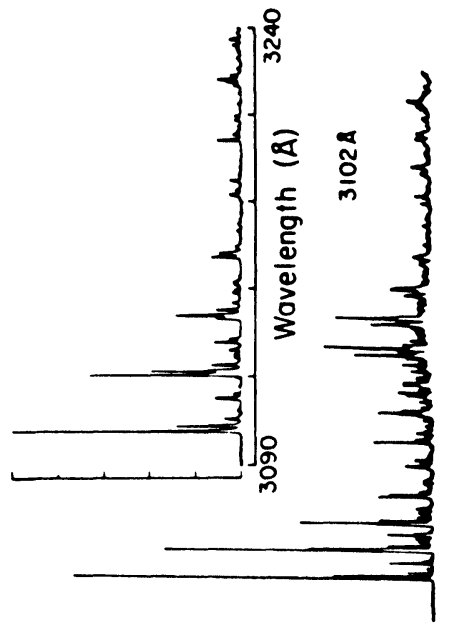

อ
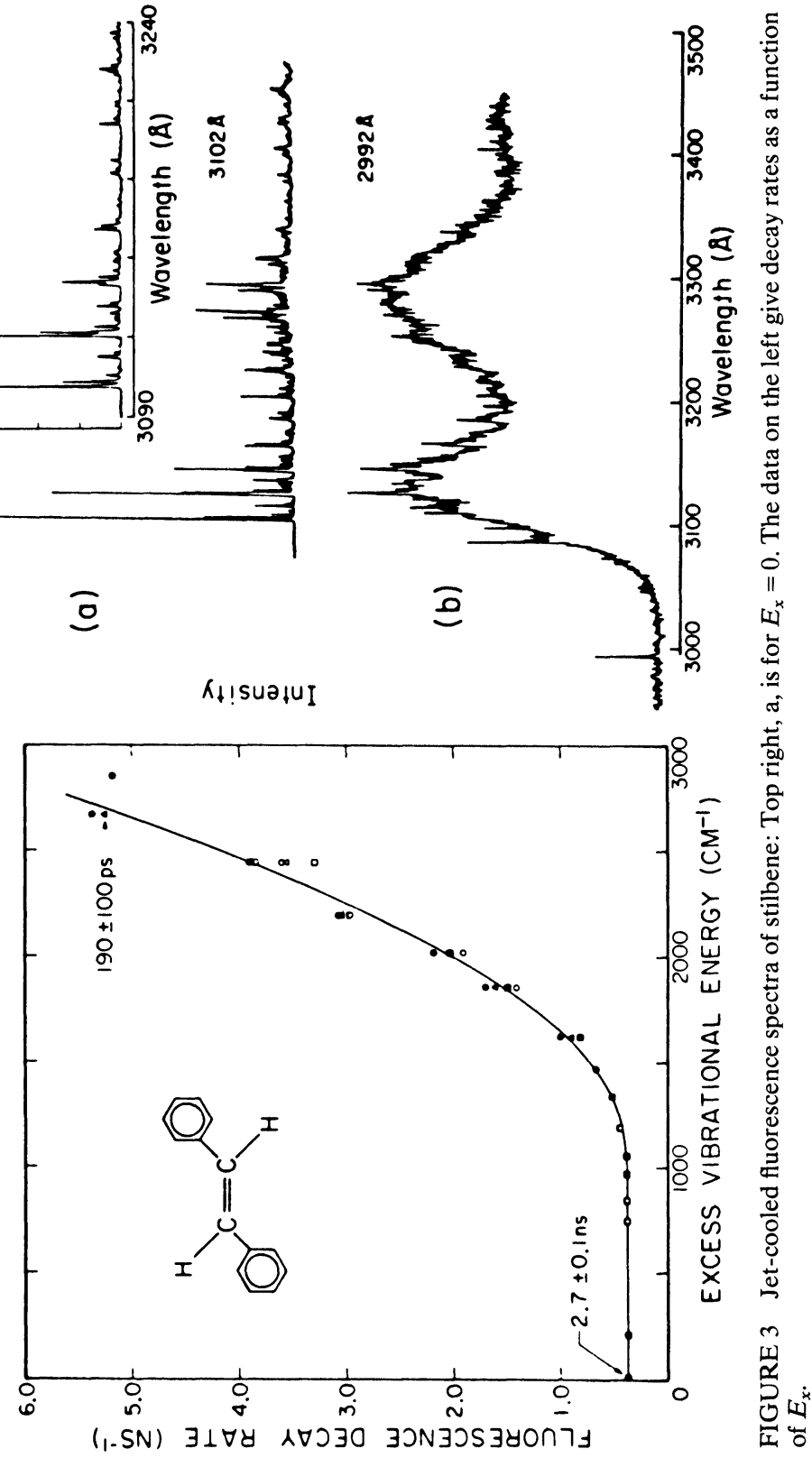
where $\bar{n}$ is the average number of modes (restricted number of modes) that the molecule "heats up" in the redistribution. If this is a real heating process, the analogy with solids for the use of specific-heat will give an effective number of modes. When we plotted $\ln k$ vs. $1 / E_{x}$ for stilbene (see data of Figure 3), we obtained a very good straight line fit with a "preexponential" of $1.5 \times 10^{11} \mathrm{~S}^{-1}$, and an apparent activation energy of $\sim 9000 \mathrm{~cm}^{-1}$ (Figure 4). Since $E_{0}$ is known from our work to be $\sim 1000 \mathrm{~cm}^{-1}$, which is in good agreement with solution phase studies of the isomerization rate, $\bar{n}$ is about 9 . This number is in surprisingly good agreement with the above "naive" prediction and with the number of low frequency modes $\left(<500 \mathrm{~cm}^{-1}\right)$ which are well-known for stilbene.

Using the classical RRK expression,

$$
k=\nu\left(\frac{E-E_{0}}{E}\right)^{S-1}
$$

we can fit the stilbene data provided $S \sim 6$ and $E_{0}$ is again in the range of $1000 \mathrm{~cm}^{-1}$, as shown in Figure 5 . One can actually show that this RRK expression can be written in an exponential form.

In a collaborative effort with $\mathrm{R}$. Marcus, we used real quantum calculations of the density of states to obtain the actual rates that are in accord with the RRKM theory. We have found the following: First, it can be shown that the $E_{0}$ obtained from our experiments ( $k$ vs. $E_{x}$ ) can be correlated with that obtained from solution phase studies $\left(k \sim A e^{-E_{0} / k T}\right)$. Second, deviations from RRKM can be handled by using the appropriate modification in rates in order to suit these new observations. These modifications include reversible rate processes for the isomerization at these excess energies and trapping of energy in certain coordinates in the transition state configuration. Comparison with RRKM calculation is shown in Figure 6, and the potential energy surface for isomerization in Figure 7.

In conclusion, the measurements of rates $v s E_{x}$ in the stilbene beam provide a way for obtaining the threshold energy ("solvent free"), and the redistribution among modes. It is possible that $B$-type modes are the main constituents of the phase space for the redistribution, and that this effect is almost universal. It is interesting that the effective temperature idea for $k$ vs. $E_{x}$ will predict that at $\sim 2000 \mathrm{~cm}^{-1}$ of excess energy, the effective temperature is approaching room 


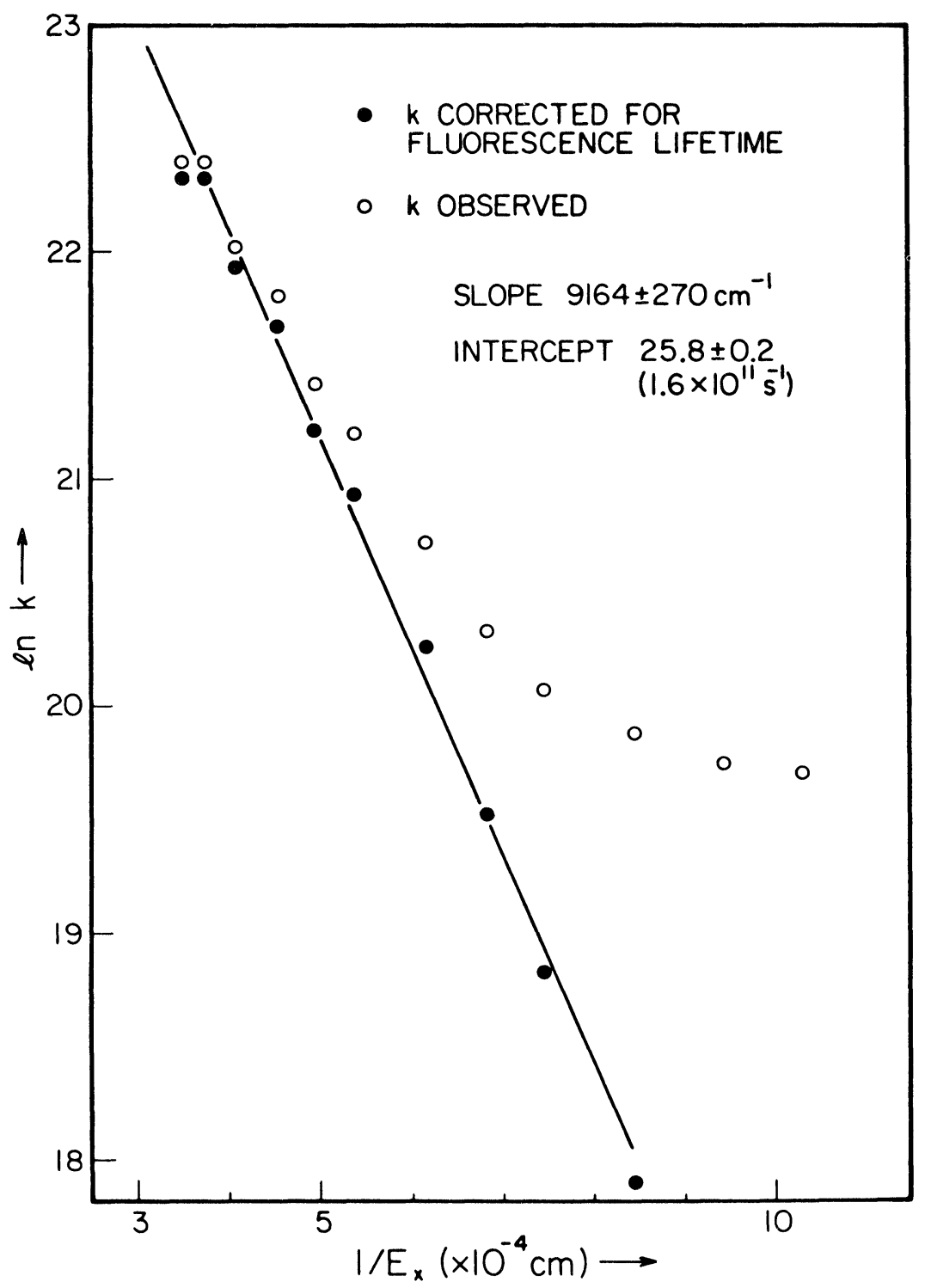

FIGURE 4 Plot of $\ln k$ (from the data of Figure 3) vs. $E_{x}^{-1}$. The open circles are the data points before correcting for the radiative decay at $E_{x}=0$, and the solid circles are after correction. 


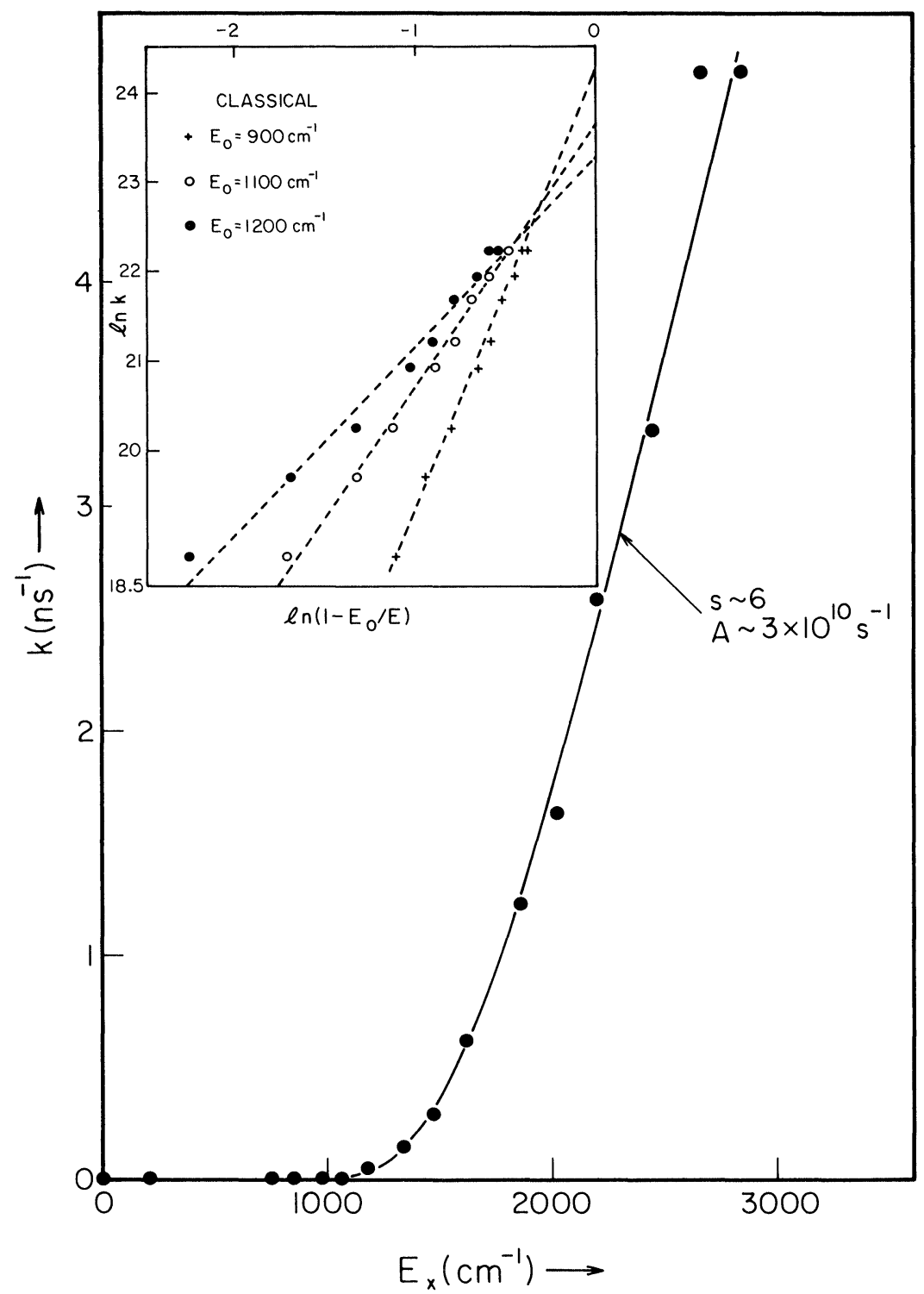

FIGURE 5 Plot of $k$ vs. $E_{x}$ for the stilbene case, together with the calculated values according to RRK theory (see text). 


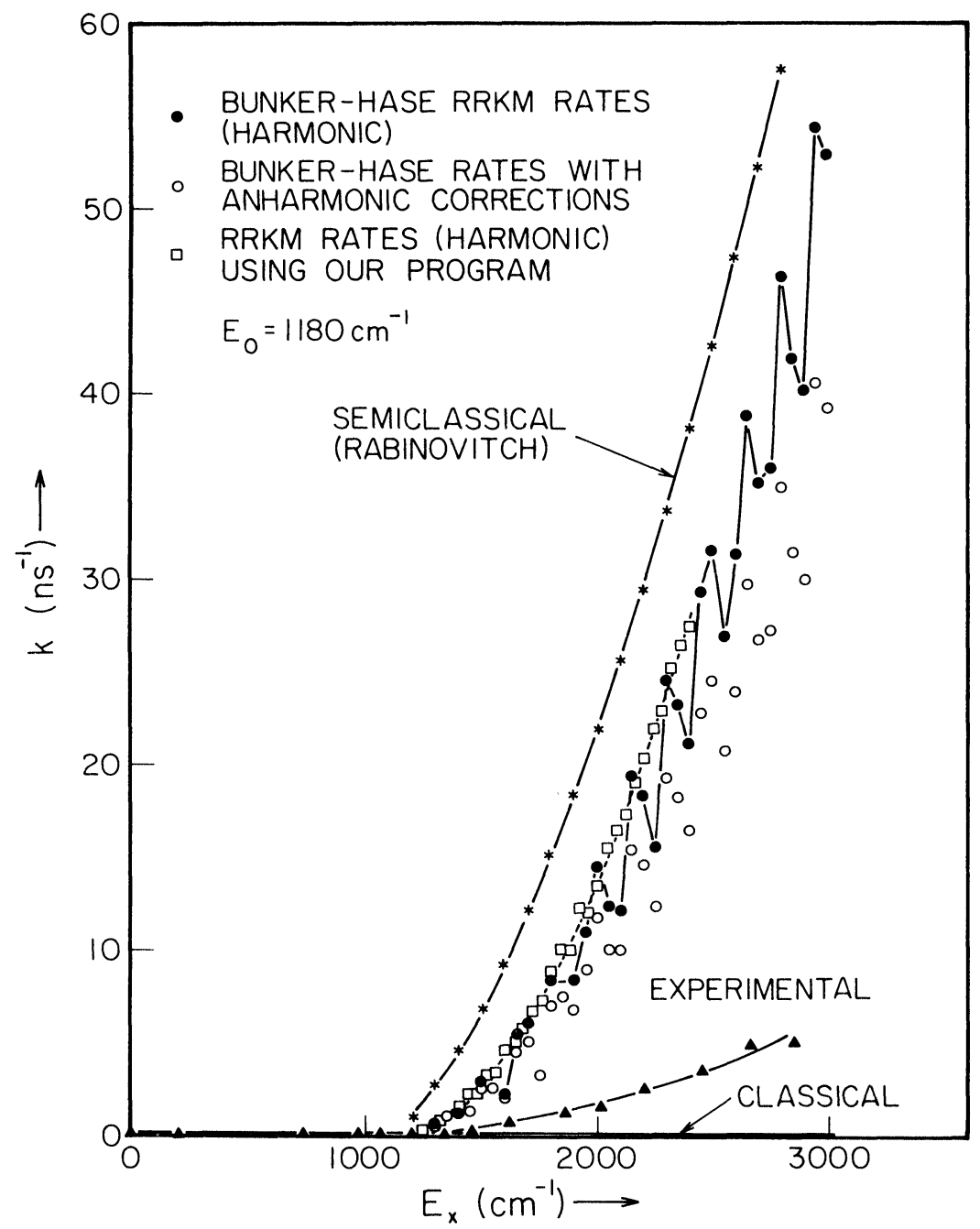

FIGURE 6 Plot of $k$ vs. $E_{x}$ for the stilbene case, together with the calculated values according to RRKM theory using different methods for calculating the density of states.

temperature, consistent with redistribution and congestion of spectra. These studies raise interesting new questions regarding these possibilities and more experiments are in progress for the test of these ideas. 


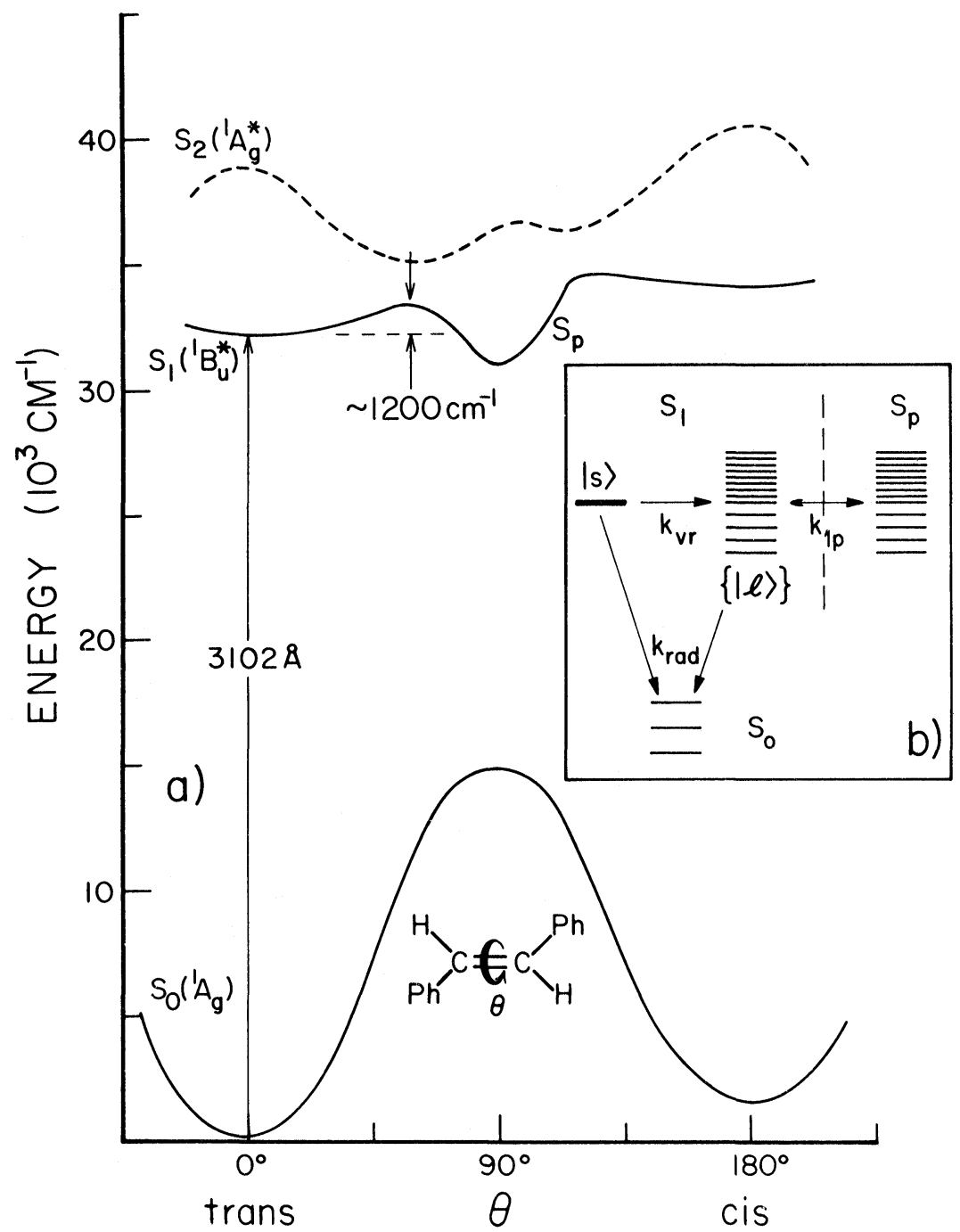

FIGURE 7 The potential energy surface proposed for isomerization. The insert describes the energy levels involved: $|s\rangle$ is optically excited, $\{|l\rangle\}$ are the levels occupied after redistribution and $S p$ are those of a twisted configuration or intermediate. 


\section{CHROMOPHORE-SELECTIVE PICOSECOND EXCITATION AND ENERGY TRANSFER}

The idea of this experiment is to excite a single chromophore in a molecule (jet-cooled) and to observe real energy flow from the optically-pumped vibrations of this chromophore to other modes of the rest of the molecule. We chose the following molecule:

$$
A^{*}-\mathrm{CH}_{2}-\mathrm{CH}_{2}-\mathrm{CH}_{2}-\phi
$$

where $A^{*}$ stands for excited anthracene and $\phi$ for an aniline moiety. This molecule was chosen because we already knew a great deal about the redistribution problem in the "optically active" chromophore, anthracene, and also because this type of molecule has been extensively studied in solutions. ${ }^{19}$ In the jet we excited the different modes of the anthracene moeity with picosecond pulses and followed the time-resolved dispersed fluorescence of anthracene-like emission and product emission (red shifted emission due to the folding of the aniline moeity towards anthracene, CT or exciplex emission; henceforth referred to as "product"). At a given excess energy, we observed a decay of the anthracene-like emission, and a buildup in the product emission (Figure 8). These rates were found to be very sensitive to the excess vibrational energy in the anthracene moeity! Thus, for the first time, one can obtain the real-time measurements of product formation due to energy flow in an isolated large molecule.

Since the conformation change needed to give the red emission requires that the vibrational modes of the propyl linkage become populated, our results indicate that the excess vibrational energy in the anthracene moeity redistributes to vibrations (and "rotations") in the side chain, thus triggering the temporal behavior of the molecule. The threshold for the excess energy dependence of the product formation is $\simeq 1000 \mathrm{~cm}^{-1}(2.9 \mathrm{kcal} / \mathrm{mol})$, consistent with a reaction barrier involving $\mathrm{C}-\mathrm{C}$ type bonds (see Figure 8). Finally, as expected, the decay and buildup time constants at $\sim 3000 \mathrm{~cm}^{-1}$ of excess energy $(\sim 400 \mathrm{ps})$ are much different from the decay time constant of bare anthracene at similar excess energies $(5.7 \mathrm{~ns})$. The question then is: what does this rate in the isolated molecule mean and what is the role of the solvent? 


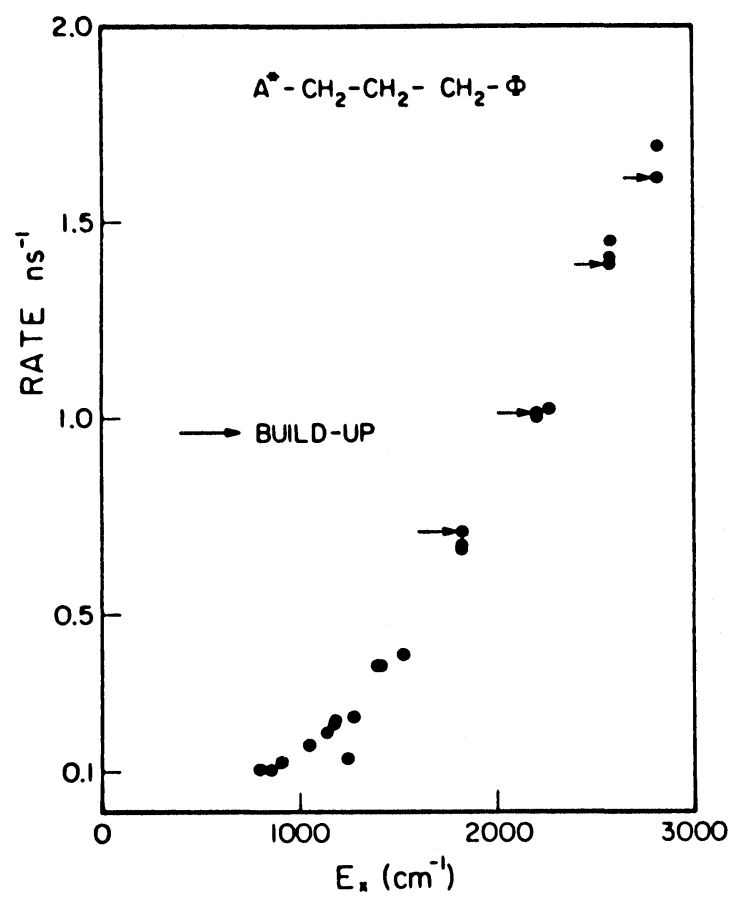

(a)

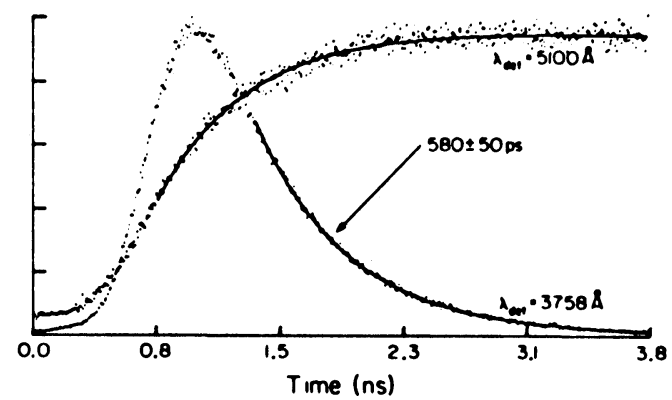

(b)

FIGURE 8 Threshold effect for rates vs. $E_{x}$ and the jet-cooled fluorescence spectra for different $E_{x}$ 's. (A) fluorescence at $E_{x}=1400 \mathrm{~cm}^{-1}$; (B) fluorescence for $E_{x}=$ $2800 \mathrm{~cm}^{-1}$; (C) fluorescence for $E_{x}=2800 \mathrm{~cm}^{-1}$ but when the spectrum was gated at short time after the picosecond pulse. Top spectrum gives the decay of the anthracenelike emission and buildup of the product (folded configuration) emission-both are identical (b). 


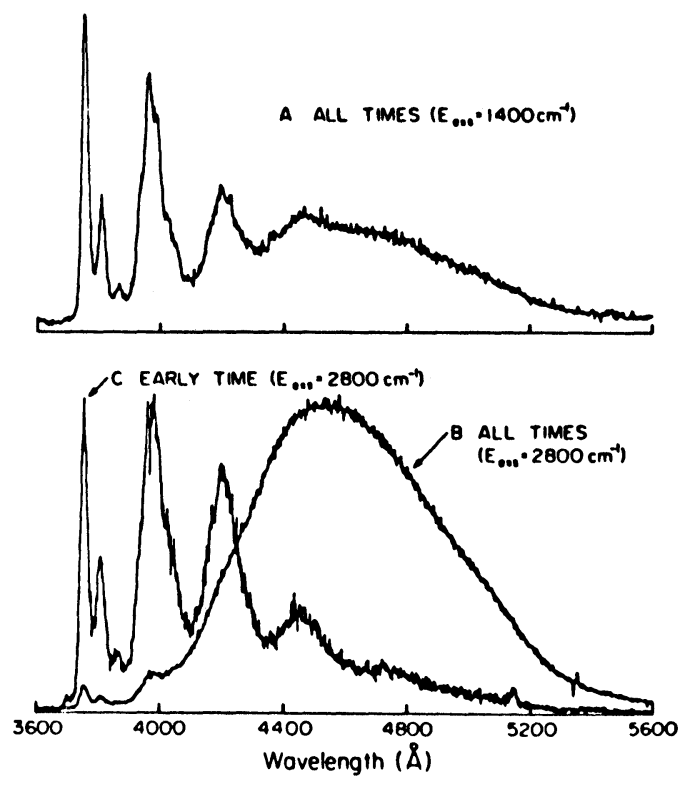

(c)

Figure 8 (cont.)

Using an assumed set of vibrational frequencies for the molecule along with our jet lifetimes at difference $E_{x}$, we have calculated the decay of the anthracene-like emission for a thermal distribution at $298 \mathrm{~K}$. The calculated lifetime ( $\sim 600 \mathrm{ps})$ is appreciably shorter than actually observed in cyclohexane solution $(1.4 \mathrm{~ns}) .{ }^{19}$ Thus, it appears that the geometrical changes needed for product formation are hindered by the solvent.

Marcus and Noyes ${ }^{20}$ have discussed the effect of diffusion on observed reaction rates. Near steady state, $\tau_{\text {obs }} \sim \tau_{\text {activ }}+\tau_{\text {diff. }}$ Consequently, the real $\tau_{\text {act }}$ can be shorter than that observed in solution. Since the diffusion coefficient is on the order of a few times $10^{-5} \mathrm{~cm}^{2} / \mathrm{sec}$, it is perhaps not surprising for a diffusion distance of order of $10 \AA$ that $\tau_{\text {diff }} \sim 1 \mathrm{~ns}$, and $\tau$ isolated molecule $\sim 500 \mathrm{ps}$. More tests of these ideas are in progress. The important point, however, is that the product formation, involving the propyl linkage, in the isolated molecule has a threshold at $\sim 1000 \mathrm{~cm}^{-1}$, and that the rate of product buildup is the same as that of the anthracene moeity decay. 


\section{STEPWISE SOLVATION AND PHOTODISSOCIATION IN THE JET}

Last year we extended the application of the picosecond-jet-technique to the study of the dynamics of isolated molecules in various stages of solvation with various solvents (water, alcohol, etc.). ${ }^{8,9,21}$ The idea was to study this "controlled" solvation and its dependence on the energy redistribution. Also, we wanted to examine the photodissociation of these different solvated species or complexes following selective pumping by the picosecond laser. The systems we studied in some detail are azine-solvent complexes made in the jet with $\mathrm{He}$ or Ar as the carrier gas.

From the dispersed fluorescence, excitation spectra and their dependence on solvent concentration, we identified the different solvated species, and obtained the frequency of the new vibrational modes that result from complexation (typically $170 \mathrm{~cm}^{-1}$ and below). For isoquinoline (IQ), three solvents were used (water, methanol and acetone) to deduce some particular effects regarding the nature of hydrogen bonding in the species:

$$
=\mathrm{N}: \cdots(\mathrm{HOR})(\mathrm{HO}-\mathrm{R})_{n}
$$

Figures 9 and 10 display typical results for the effect of solvent on the excitation spectra, fluorescence and lifetimes. We used these observations to measure bond dissociation energy of the different species by varying the excess vibrational energy in the "parent" molecule type modes. The threshold effect found for the dissociation of the 1:1 IQ (methanol and water) is evident (Figure 11) and occurs at $\sim 3 \mathrm{kcal} / \mathrm{mol}$. (The effect of higher degree of solvation on the dynamics will be discussed elsewhere).

The breakage of the hydrogen bond in these solvated species probes two interesting dynamical effects: the photodissociation of the solvent and the vibrational energy redistribution from the bare molecule (IQ) type modes to the dissociation coordinate. In this system, the solutesolvent complex at zero excess vibrational energy has a much longer lifetime than the bare solute molecule. However, above the barrier the complex has a much shorter lifetime (approaching the bare molecule) than that at $E_{x}=0$. At the barrier energy the density of low-frequency modes is enormous due to hydrogen bonding. Using the RRKM calculation (see previous section) we can fit these data 

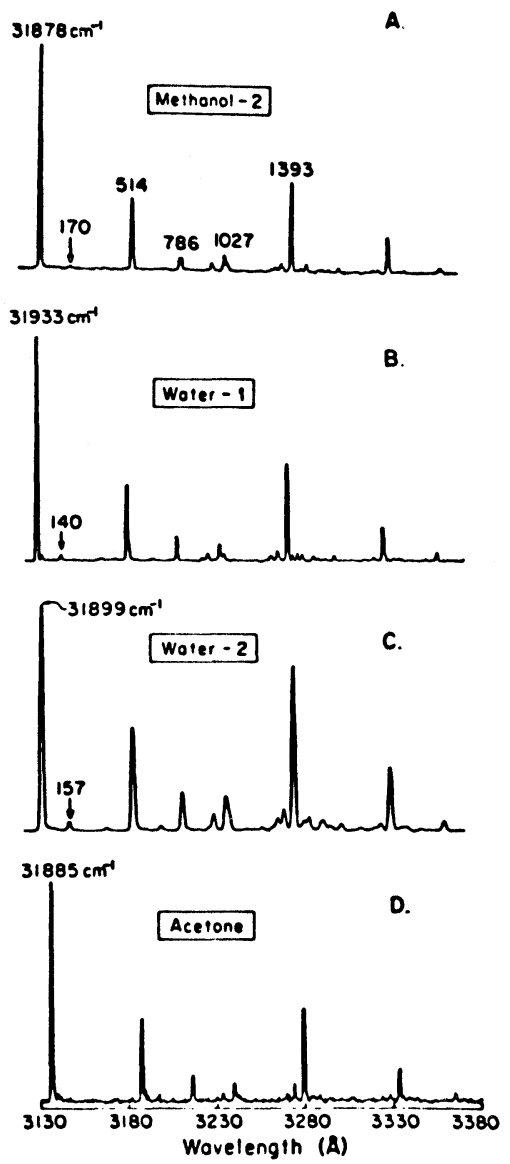

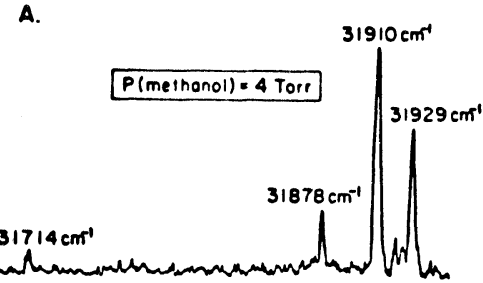

B.
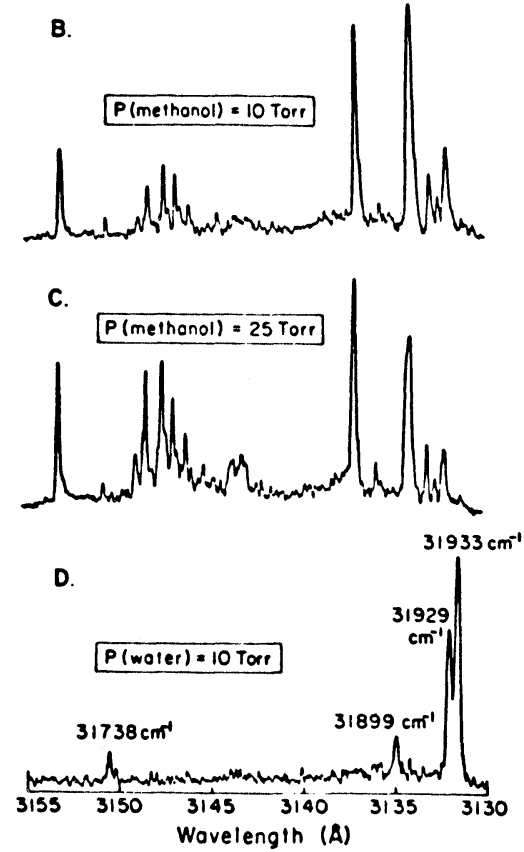

FIGURE 9 Jet-cooled fluorescence spectra of IQ-solvent (left) and excitation spectra (right) at different solvent concentrations. (a)-(c) on the right give excitation spectra at 4,10 and 25 torr methanol, respectively. (d) is the spectrum at 10 torr water. On the left, (a) is for methanol-2 species, (b) and (c) for water-1 and water-2 species, and (d) for acetone. Details are given in the references quoted in the text.

quite well (Figure 11) and invoke the statistical nature of energy redistribution. This is not surprising given the very high density-ofstates. How does the cluster size influence the redistribution is currently under investigation. 


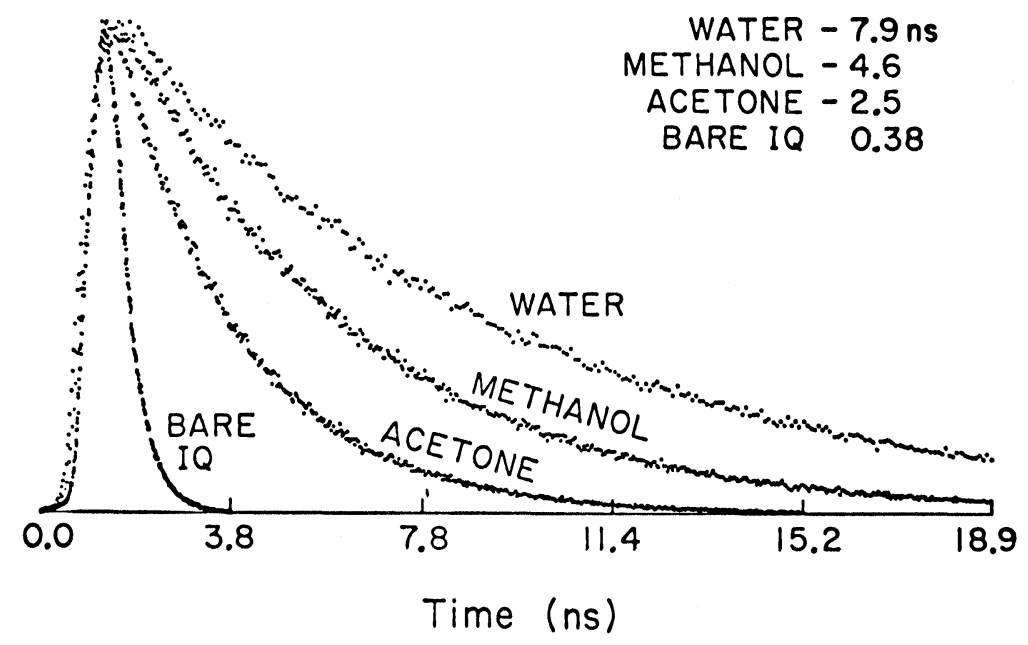

FIGURE 10 Lifetimes of IQ-solvent complexes in the jet: bare IQ, 380 ps; acetone, $2.5 \mathrm{~ns}$; methanol, $4.6 \mathrm{~ns}$; and water, $7.9 \mathrm{~ns}$.

\section{CONCLUSIONS}

In this article we provided a description of the technique of "picosecond-jet," where a picosecond pulse crosses a supersonicallycold beam of large molecules. The chemistry and spectroscopy done in this laboratory for a number of systems were reviewed, but no background materials were given for details on the importance of jet excitation and fluorescence spectroscopy. ${ }^{22,23}$ Our studies include: isomerization, vibrational energy transfer, quantum beats and partial solvation of large molecules in jets.

Finally, by varying the excess vibrational energy in the molecule we were able, from real-time measurements, to obtain selective rate constants $k(m, E)$ which exhibit a threshold effect. Comparison with theory was made to access the role of energy redistribution: statistical or nonstatistical. We hope to report soon on the application of the technique to molecules exhibiting fast energy redistribution at room temperature, as shown by the experiments of Parmenter. ${ }^{13}$ 

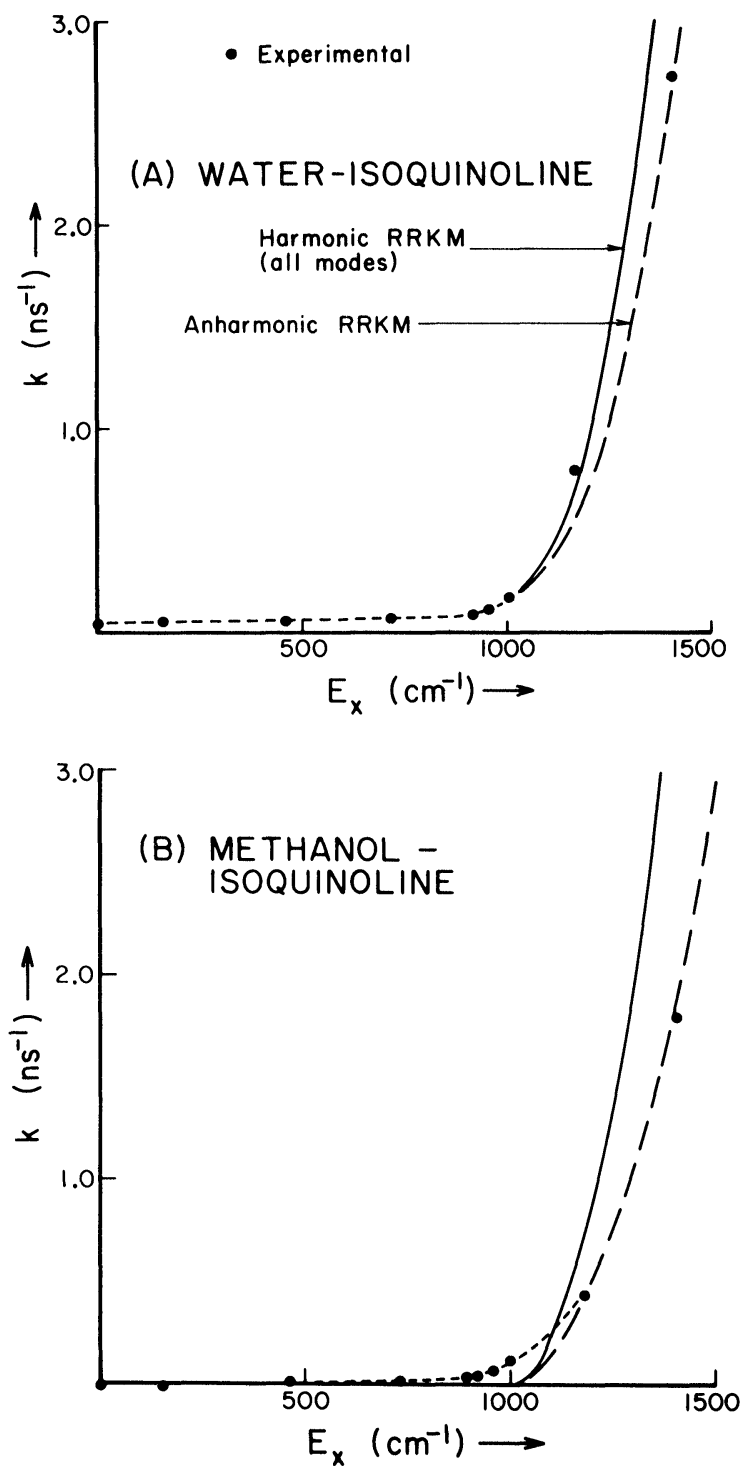

FIGURE 11 Decay rates, $k$, vs. $E_{x}$ for IQ-water and IQ-methanol complexes, together with the calculated RRKM rates for the bond breakage of the hydrogen bonded systems. Note the magnitude of the threshold energy which is consistent with H-bond energy. 


\section{Acknowledgements}

This work was supported by grants from the National Science Foundation (CHE8112833 and DMR8105034). I would like to thank Professors R. Marcus and J. Hopfield for enlightening discussions, and members of my research group who worked with dedication to make this research possible.

\section{References}

1. Wm. R. Lambert, P. M. Felker and A. H. Zewail, J. Chem. Phys. 75, 5958, (1981).

2. A. H. Zewail, in: Picosecond Phenomena III, eds. K. Eisenthal, R. Hochstrasser, W. Kaiser and A. Laubereau (Springer-Verlag, New York, 1982) p. 184.

3. J. A. Syage, Wm. R. Lambert, R. M. Felker, A. H. Zewail and R. M. Hochstrasser, Chem. Phys. Lett. 88, 266 (1982); Wm. R. Lambert, P. Felker and A. H. Zewail, to be published.

4. P. M. Felker, Wm. R. Lambert and A. H. Zewail, Chem. Phys. Lett. 89, 309 (1982).

5. J. Syage, P. Felker and A. H. Zewail, to be published.

6. P. M. Felker, Wm. R. Lambert and A. H. Zewail, J. Chem. Phys. 77, 1603 (1982); P. M. Felker and A. H. Zewail, to be published.

7. P. M. Felker, J. A. Syage, Wm. R. Lambert and A. H. Zewail, Chem. Phys. Lett. 92, 1 (1982).

8. P. M. Felker and A. H. Zewail, Chem. Phys. Lett. 94, 448, (1983).

9. P. M. Felker and A. H. Zewail, Chem. Phys. Lett. 94, 454 (1983).

10. D. Millar and A. H. Zewail, Chem. Phys. 72, 381 (1982).

11. See the article by J. Kommandeur (and references therein) in this volume.

12. B. Keelan and A. H. Zewail, in preparation.

13. See the article by C. Parmenter in this volume.

14. R. Sharp, E. Yablonovitch and N. Bloembergen, J. Chem. Phys. 76, 2147 (1982).

15. See, e.g., J. Saltiel and J. Charlton, in: Organic Chemistry, (Academic Press, New York, 1980) Vol. 42-3, p. 25.

16. R. M. Hochstrasser, Pure Appl Chem. 52, 2683 (1980).

17. A. H. Zewail, in Physics Today 33, 27 (1980).

18. N. Bloembergen and E. Yablonovitch, Phys. Today 31, 23 (1978).

19. See the article by $\mathbf{K}$. Eisenthal in this volume, and references therein.

20. R. A. Marcus, Faraday Discuss, Chem. Soc. 29, 129 (1960); R. M. Noyes, Prog. React. Kinet. 1, 129 (1961).

21. P. M. Felker and A. H. Zewail, J. Chem. Phys. 78, 5266 (1983).

22. For a review, see, e.g., the article by D. H. Levy in Photoselective Chemistry, eds. J. Jortner, R. Levine and S. Rice (Wiley Interscience, New York, 1981), and ref. 23.

23. A. Amitav, U. Even and J. Jortner, J. Chem. Phys. 86, 3345 (1982); and references therein. 